\title{
ESTUDO ANATÔMICO DA MADEIRA DE CITHAREXYLUM MONTEVIDENSE MOLDENKE (VERBENACEAE) ${ }^{1}$
}

\author{
ANELISE MARTA SIEGLOCH² JOSÉ NEWTON CARDOSO MARCHIORI ${ }^{3}$ \\ SIDINEI RODRIGUES DOS SANTOS ${ }^{4}$
}

\begin{abstract}
RESUMO
É anatomicamente descrita a madeira de Citharexylum montevidense Moldenke, com base em material coletado no município de Pinheiro Machado, Rio Grande do Sul. A madeira apresenta: vasos de diâmetro pequeno, solitários e em curtos múltiplos radiais; elementos vasculares de comprimento médio, com apêndices; placas de perfuração simples; pontoações intervasculares de diâmetro médio, alternas e ornamentadas; parênquima paratraqueal-escasso; raios heterogêneos; e fibras libriformes septadas.

Palavras-chave: Anatomia da madeira, Citharexylum montevidense, Verbenaceae.
\end{abstract}

\section{ABSTRACT}

[Wood anatomy of Citharexylum montevidense Moldenke (Verbenaceae)].

The wood anatomy of Citharexylum montevidense Moldenke is described, based on sample collected in the municipality of Pinheiro Machado, Rio Grande do Sul State (Brazil). The wood structure presents: small diameter vessels; solitary and short radial multiple pores; medium length vascular elements, with appendices; simple perforation plates; medium size intervascular pits, with alternate-ornamented bordered pits; scanty paratracheal parenchyma; heterogeneous rays; and septate fibers.

Key words: Citharexylum montevidense, Verbenaceae, Wood Anatomy.

\section{INTRODUÇÃO}

De distribuição pantropical e, principalmente, neotropical, a família Verbenaceae inclui cerca de 36 gêneros e 1000 espécies (Souza \& Lorenzi, 2012). Compreende ervas, lianas, arbustos e árvores, geralmente com caules quadrangulares e, por vezes, providos de acúleos ou espinhos (Judd et al., 2009).

Entre os principais gêneros representados incluem-se: Verbena (200 spp.), Lippia (200), Lantana (150), Cytharexylum (130), Glandularia (100), Stachytarpheta (90) e

Recebido para publicação em 14/06/2013 e aceito para publicação em 5/09/2013.

2 Mestranda do Curso de Pós-Graduação em Engenharia Florestal. Bolsista - CAPES. Universidade Federal de Santa Maria, CEP 97105-900. Santa Maria, RS, Brasil. anesiegloch@yahoo.com.br

3 Engenheiro Florestal, Dr. Bolsista de Produtividade em Pesquisa (CNPq - Brasil). Professor Titular do Departamento de Ciências Florestais, Universidade Federal de Santa Maria. Santa Maria, RS, Brasil.

${ }^{4}$ Biólogo, Dr. Núcleo de Estudos Botânicos Balduino Rambo, UFSM.
Duranta (20) (Judd et al., 2009). Na flora arbórea do Rio Grande do Sul, o gênero mais expressivo é Citharexylum, com três espécies: C. montevidense, C. myrianthum e $C$. solanaceum (Sobral et al., 2006). Citharexylum montevidense, objeto do presente estudo, ocorre naturalmente na Argentina, Paraguai, Uruguai e sul do Brasil (Troncoso, 1979), sendo especialmente comum em florestas da metade meridional do Rio Grande do Sul (Sobral et al., 2006).

Produtora de madeira, Citharexylum montevidense pode alcançar $15 \mathrm{~m}$ de altura, apresentando fuste reto e casca cinza-esverdeada com finas estrias longitudinais. As folhas, simples, opostas e decussadas, são coriáceas, de lâminas espatuladas, obovadas, oblongas ou elípticas (2-4 x 5-8,5 cm), com nervura central proeminente e indumento na axila das nervuras secundárias. As flores, dispostas em racemos espiciformes axilares e terminais, aparecem ao final da primavera e são muito perfumadas (Brussa \& Grella, 2006). 
Com relação à anatomia do lenho, Metcalfe \& Chalk (1972) relacionam os seguintes caracteres para as Verbenaceae: vasos de diâmetro médio, em padrão radial, oblíquo ou tangencial; porosidade comumente em anel; elementos vasculares de comprimento médio a muito curto; placas de perfuração simples, com algumas escalariformes e/ou reticuladas em alguns gêneros; pontoações intervasculares diminutas a moderadamente grandes; pontoações raio-vasculares, geralmente semelhantes; parênquima paratraqueal estreito-vasicêntrico, por vezes abundante e confluente; raios heterogêneos a homogêneos, com 3-4 células de largura, por vezes muito largos; e fibras com pontoações simples, geralmente septadas, de comprimento médio a moderadamente curto.

$\mathrm{Na}$ literatura de Verbenaceae são citados diversos tipos de placas de perfuração: simples, raro escalariformes e reticuladas, ou combinação de placas simples até escalariformes (Record \& Hess, 1949; Metcalfe \& Chalk, 1972, Meylan \& Butterfield, 1975). Para o gênero Citharexylum foram descritas: placas de perfurações predominantemente simples, com placas radiadas e foraminado-radiadas, em $C$. myrianthum Cham. (Gomes et al., 1989); mistura de placas tipicamente reticuladas e foraminado-reticuladas, em $C$. fruticosum (Gottwald \& Parameswaram, 1964); e placas simples (maioria) e multiperfuradas, dos tipos escalariforme, irregularmente reticulada, foraminado-radiada e radiada, em $C$. solanaceum Cham. (Gomes et al., 2008).

O presente estudo visa a descrição da madeira de Citharexylum montevidense e identificar caracteres de valor taxonômico, comparando-os com referências da literatura.

\section{MATERIAL E MÉTODOS}

O material em estudo consiste de uma amostra de madeira e respectivo material botânico, conservados na Xiloteca do Herbário do Departamento de Ciências Florestais (HDCF) da Universidade Federal de Santa Maria com os se- guintes registros: Citharexylum montevidense Moldenke, Pedras Altas (Pinheiro Machado, RS), HDCF 1781.

Para a confecção das lâminas histológicas foram extraídos três corpos de prova $(1 \times 2 \times 3 \mathrm{~cm})$ da parte mais externa do lenho, próxima ao câmbio, orientados para a obtenção de cortes nos planos transversal, longitudinal radial e longitudinal tangencial. Outro bloquinho foi também retirado, com vistas à maceração.

A confecção das lâminas histológicas seguiu a metodologia descrita em Burger \& Richter (1991). No preparo de lâminas de macerado usou-se o método de Jeffrey (Freund, 1970). Os cortes anatômicos foram tingidos com acridinavermelha, crisoidina e azul-de-astra (Dujardin, 1964); o macerado, apenas com safranina (1\%). Na montagem das lâminas permanentes usouse Entellan.

As descrições basearam-se nas recomendações do IAWA Committee (1989). No caso da percentagem dos tecidos foram realizadas 600 determinações ao acaso, com auxílio de contador de células, conforme proposto por Marchiori (1980). A abundância de poros foi obtida a partir de um quadrado de área conhecida, superposto a fotomicrografias de seções transversais da madeira.

As medições foram realizadas em microscópio Carl Zeiss, no Laboratório de Anatomia da Madeira da Universidade Federal de Santa Maria. Nas características quantitativas, os números entre parênteses equivalem aos valores mínimos e máximos observados; o valor que acompanha a média é o desvio padrão. As fotomicrografias foram tomadas em microscópio Olympus CX40, equipado com câmera digital Olympus Camedia c3000, no Laboratório de Anatomia da Madeira da Universidade Federal do Paraná.

\section{DESCRIÇÃO ANATÔMICA}

Anéis de crescimento: distintos, evidenciados pelo aspecto anelar, produzido pelos poros menores no lenho tardio e de maior diâmetro 

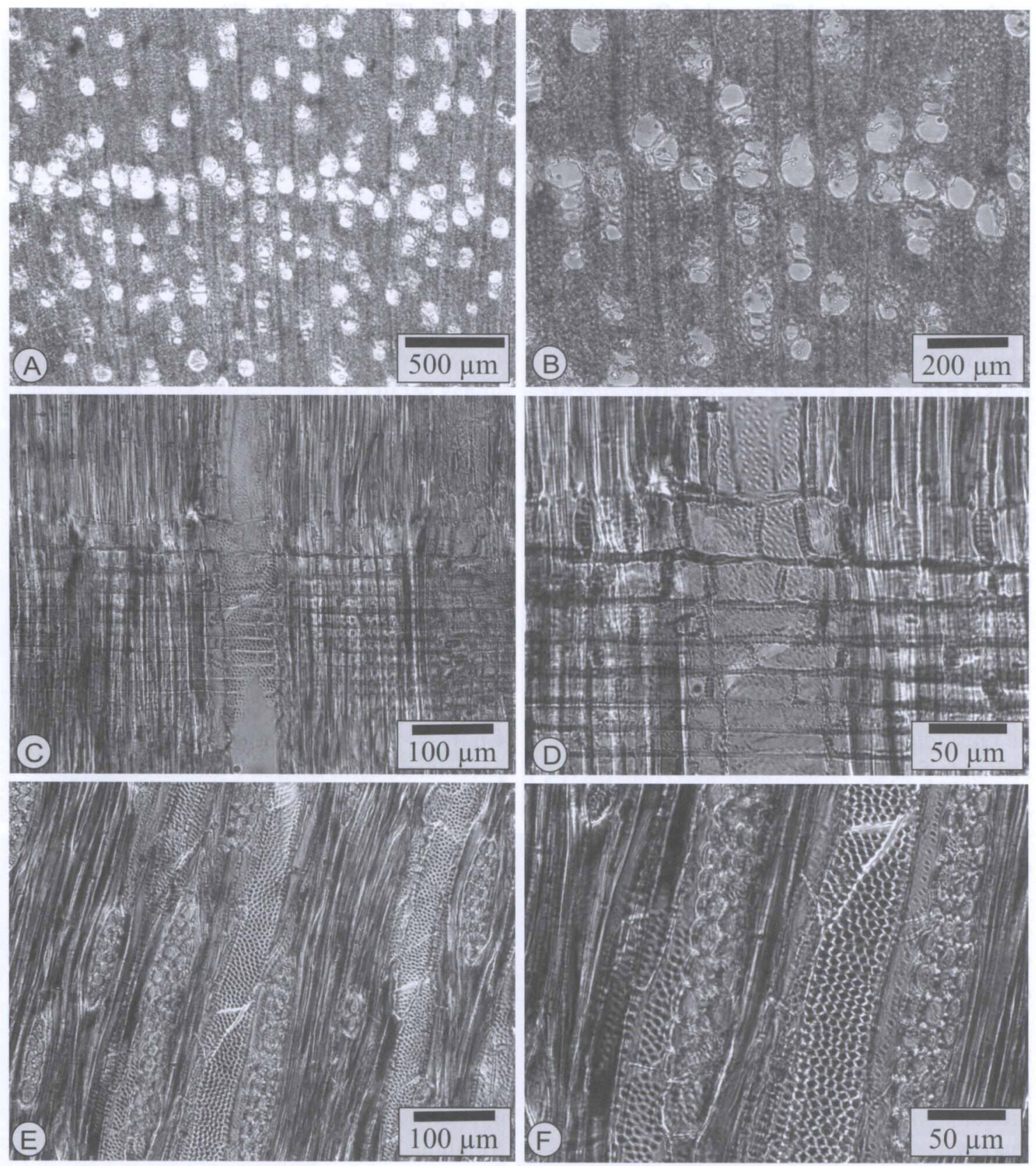

FIGURA 1 - Aspectos anatômicos da madeira de Citharexylum montevidense Moldenke. A-Limite de anel de crescimento, porosidade semi-difusa e poros solitários (seção transversal). B - Limite do anel de crescimento, delimitado por poros de maior diâmetro, fibras achatadas e parênquima marginal (seção transversal). C - Fibras septadas, raio heterogêneo, com células procumbentes no corpo central e células eretas e/ou quadradas nas margens (seção longitudinal. radial). D - Seção longitudinal radial, destacando parte de raio com células procumbentes e eretas. E - Raios uni a trisseriados, elementos vasculares com placas de perfuração transversais e fibras septadas (seção longitudinal radial). F - Vasos com pontoações intervasculares alternas, placa de perfuração transversal, raios trisseriados, parênquima axial paratraquealescasso e fibras septadas (seção longitudinal tangencial). 
no lenho inicial, bem como pela diferença na espessura da parede de fibras e presença de parênquima marginal (Figura $1 \mathrm{~A}, \mathrm{~B}$ ).

Vasos: muito numerosos $(43 \pm 6(36-50)$ poros $/ \mathrm{mm}^{2}$ ), ocupando $17 \pm 2,6 \%$ do volume da madeira. Porosidade semi-difusa, tendente a anelar. Poros solitários e em curtos múltiplos radiais (2-3); circulares ou ovais $(70 \pm 13,8$ (40 - 95) $\mu \mathrm{m})$ e de paredes finas $(2,8 \pm 0,8(1,3-$ 3,8) $\mu \mathrm{m}$ (Figura $1 \mathrm{~A}, \mathrm{~B})$. Elementos vasculares de comprimento médio (400 $\pm 59(300-530)$ $\mu \mathrm{m})$, com apêndices em uma ou em ambas as extremidades $(20-150 \mu \mathrm{m})$ e finas estriações helicoidais na parede. Placas de perfuração simples (Figura 2F), por vezes tipicamente foraminadas (Figura 2D), foraminado-radiadas (Figura 2 C,E) e múltiplo-radiadas (Figura 2 A,B), oblíquas ou transversais. Pontoações intervasculares pequenas, circulares $(5,8 \pm 0,5$ $(5,2-6,2) \mu \mathrm{m})$, alternas, com abertura em fenda elíptica, inclusa, ornamentada. Pontoações raio-vasculares semelhantes às intervasculares, porém menores $(4,9 \pm 0,4(4-5,2) \mu \mathrm{m})$ (Figura 1 C,D). Conteúdos, ausentes.

Parênquima axial: marginal (limite do anel de crescimento) e paratraqueal-escasso, representando $5 \pm 2,3 \%$ do volume da madeira ( $\mathrm{Fi}$ gura 1 A,B). Séries parenquimáticas de $341 \pm$ 48 (237 - 450) $\mu \mathrm{m}$ de altura, compostas de $2-4$ células (Figura $1 \mathrm{E}, \mathrm{F}$ ).

Raios: numerosos $(7 \pm 1,7(6-10)$ raios/ $\mathrm{mm}$ ), heterogêneos, com 1-3 células de largura (Figura $1 \mathrm{E}, \mathrm{F}$ ), ocupando $15 \pm 3,6 \%$ do volume da madeira (Figura 1 A,B). Os unisseriados, de $120 \pm 34,4(60-230) \mu \mathrm{m}$ e $2-5$ células de altura. Os multisseriados, de $296 \pm 81(180-500)$ $\mu$ m e 9 - 24 células de altura, com células procumbentes no corpo central e 1-2 fileiras marginais de células quadradas e/ou eretas (Figura 1 C,D). Raios fusionados, presentes. Conteúdos, células radiais de paredes disjuntas, células perfuradas, raios agregados, cristais e células envolventes, ausentes.

Fibras: libriformes, septadas (1-3 septos/fibras $)$; de $1072 \pm 142(830-1370) \mu \mathrm{m}$ de comprimento, com $19 \pm 2(15-22,5) \mu \mathrm{m}$ de largu- ra, e paredes finas a espessas $(4,8 \pm 0,8(3,7-$ $6,3) \mu \mathrm{m}$ ), ocupando $63 \pm 3,6 \%$ do volume da madeira (Figura 1 A,B ). Fibras gelatinosas, presentes. Espessamentos espiralados e traqueóides, ausentes.

Outros caracteres: variantes cambiais, tubos laticíferos e taniníferos, canais intercelulares, células oleíferas, inclusões minerais, células mucilaginosas, estratificação e máculas medulares, ausentes.

\section{ANÁLISE DA ESTRUTURA ANATÔMICA}

A estrutura microscópica da madeira de Citharexylum montevidense corresponde, em linhas gerais, ao referido por Metcalfe \& Chalk (1972) para Verbenaceae: vasos de diâmetro pequeno, solitários e em curtos múltiplos radiais; elementos vasculares de comprimento médio, com apêndices; placas de perfuração simples; pontoações intervasculares de diâmetro médio, alternas e ornamentadas; pontoações raio-vasculares semelhante às intervasculares; parênquima paratraqueal-escasso; raios heterogêneos; e fibras libriformes septadas.

Para o gênero Citharexylum, Metcalfe \& Chalk (1972) reportaram poros solitários e porosidade semi-difusa, e Gomes et al. (1989) descreveram porosidade semi-difusa e parênquima axial marginal para C. myrrianthum, caracteres igualmente observados na espécie estudada.

Ao contrário do observado por Metcalfe \& Chalk (1972), em C. montevidense não se encontram raios com tendência à estratificação e nem carbonato de cálcio na estrutura lenhosa.

Foram confirmados, na espécie em estudo, alguns dos tipos de placas de perfuração referidos na literatura, caso das placas tipicamente foraminadas, foraminado-radiadas e múltiploradiadas. Quanto a este aspecto, vale ressaltar que as placas de perfuração ganham nitidez com a microscopia eletrônica de varredura, embora sejam passíveis de detecção por microscopia fotônica. Segundo Gomes et al. (1989), o termo múltiplo-radiado consta apenas para o gênero Citharexylum, embora tenha sido registrado por 

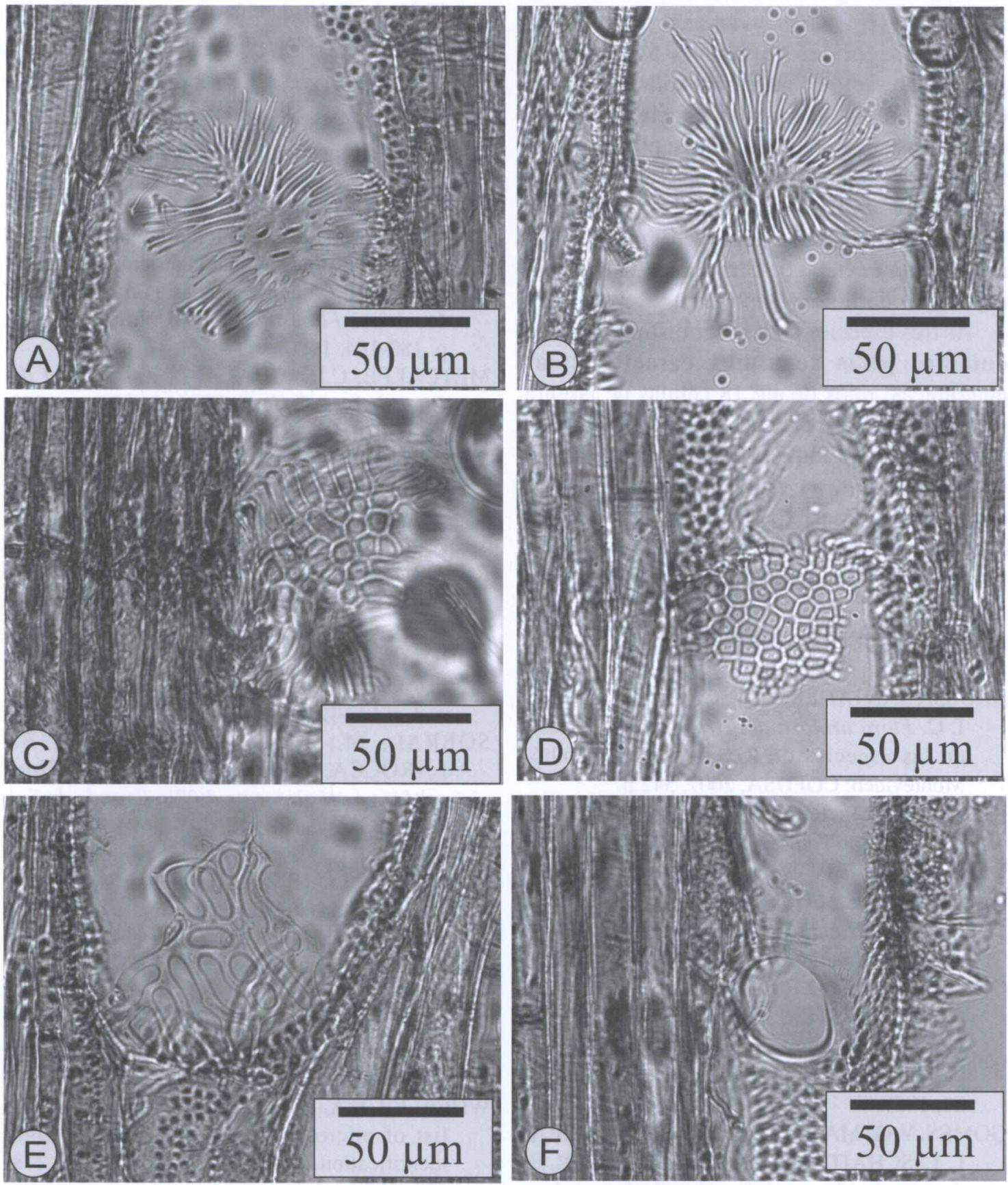

FIGURA 2 - Placas de perfuração no lenho de Citharexylum montevidense Moldenke, em seções longitudinais radiais. A,B - Placas de perfuração múltiplo-radiadas. C - Placa de perfuração foraminado-radiada. D - Placa de perfuração tipicamente foraminado. E - Placa de perfuração foraminada. F - Placa de perfuração simples. 
Ruddall (1982) em Canthium barbatum (Rubiaceae).

Com base em Citharexylum myrianthum, $C$. solanaceum e $C$. montevidense, o gênero apresenta os seguintes caracteres anatômicos: porosidade semi-difusa; poros solitários; parede de vasos com finas estriações helicoidais; elementos vasculares com apêndices; placas de perfuração predominantemente simples, foraminadoradiadas e múltiplas radiadas; pontoações intervasculares diminutas a pequenas; parênquima axial com 2-4 células de altura; raios heterogêneos; e fibras libriformes septadas.

As três espécies podem ser distinguidas, no entanto, pelas seguintes características anatômicas: raios de até 18 células de largura (Citharexylum solanaceum); raios uni a trisseriados e parênquima paratraqueal-escasso (C. montevidense); e raios uni a tetrasseriados, com parênquima paratraqueal vasicêntrico, aliforme a confluente e curtas bandas tangenciais (C. myrianthum).

\section{REFERÊNCIAS BIBLIOGRÁFICAS}

BRUSSA SANTADER. C. A.; GRELA GOZÁLEZ. I. C. Flora arbórea del Uruguay. Con énfasis en las especies de Rivera y Tacuarembó. Montevideo: COFUSA, 2007. 542 p.

BURGER, L.M.; RICHTER, H.G. Anatomia da Madeira. São Paulo: Ed. Nobel, 1991. 154 p.

DUJARDIN, E.P. Eine neue Holz-Zellulosenfaerbung. Mikrokosmos, n. 53, p. 94, 1964.

FREUND, H. Handbuch der Mikroskopie in der Technik. Frankfurt: Umsham Verlag, 1970.375 p. GOMES, A. V.; TEIXEIRA, L. L.; SCHAITZA, E. G.; HOFMEISTER, R. M. Perforation plates in vessels of Citharexylum myrianthum Cham. (Verbenaceae). IAWA Bulletin, v.10, n.1, p. 2734, 1989.

GOMES, V. G.; MARCHIORI, J. N. C.; TEIXEIRA, L, L; SCHAITZA, E. G.; STOFELLA, D. R. E. Anatomia da madeira de Cytharexylum solanaceum Cham. (Verbenaceae). Balduinia, $\mathrm{n}$. 12, p. 13-25, 2008.

GOTTWALD, H.; PARAMESWARAM, N. Vielfache Gefassdurchbrechungen in der Familie Dipterocarpaceae. Zaitschr. F. Bot., n. 52, p. 321334, 1964.

JUDD, W. S.; CAMPBELL, C. S.; KELLOGG, E. A.; STEVENS, P. F; DONOGHUE, M. J. Sistemática vegetal: um enfoque filogenético. Porto Alegre: Artmed, 2009. p. 490-492.

MARCHIORI, J.N.C. Comprovação da viabilidade da utilização da secção longitudinal tangencial para a determinação histométrica dos elementos axiais do xilema secundário. In: Anais do IV Congresso Florestal Estadual, Nova Prata, RS, p. 180-184, 1980.

METCALFE, C.R.; CHALK, L. Anatomy of the Dicotyledons. Oxford: Clarendon Press, 1972. p. 1030-1041.

MEYLAN, B. A.; BUTTERFIELD, B. G. Occurrence of simple, multiple and combination perforation plates in the vessels of New Zealand woods. New Zeal. J. Bot., n. 13, p. 1-18, 1975.

RECORD, S. J.; HESS, R. W. Timbers of the New World. New Haven: Yale University Press, 1949. p. 436-443.

RUDDALLP. J. An unusual type of perforation plate in Canthium barbatum (Rubiaceae). IAWA Bull., n. 3, p 127-129, 1982.

SOBRAL, M.; BRACK, P.; IRGANG, B.; LAROCCA, J.; RODRIGUES, R. S. Flora arbórea e arborescente do Rio Grande do Sul, Brasil. São Carlos: RiMA: Novo Ambiente, 2006. 350 p.

SOUZA, V.C.; LORENZI, H. Botânica Sistemática: guia ilustrado para identificação das famílias de Angiospermas da flora brasileira, baseado em APG III. Nova Odessa: Instituto Plantarum, 2012. p. 674-678.

TRONCOSO, N. S. Verbenaceae. In: Burkart, A. (org.) Flora ilustrada de Entre Rios(Argentina) Buenos Aires, INTA, 1979, v.6, parte 5, p. 229-294.

WHEELER, E.A.; BAAS, P.; GASSON, P.E. IAWA list of microscopic features for hardwood identification. IAWA Bull., v.10, n. 3, p. 218359, 1989. 